\title{
Borg CR-10 scale as a new approach to monitoring office exercise training
}

\begin{abstract}
Background: There are many potential training exercises for office workers in an attempt to prevent musculoskeletal disorders. However, to date a suitable tool to monitor the perceived exertion of those exercises does not exist.

Objective: The primary objective of this study was to examine the validity and reliability of the Borg CR-10 scale to monitor the perceived exertion of office exercise training.

Methods: The study involved 105 staff members employed in a government office with an age range from 25 to 50 years. The Borg CR-10 scale was self-administered two times, with an interval of two weeks in order to evaluate the accuracy of the original findings with a retest. Face validity and content validity were also examined.

Results: Reliability was found to be high for the Borg CR-10 scale (0.898). Additionally a high correlation between the Borg CR-10 scale and Visual Analog Scale (VAS) was identified (rs = $0.754, \mathrm{P}<0.01)$.

Conclusions: This study found the Borg CR-10 scale to be a reliable and valid tool for monitoring the perceived exertion of office exercise training and may potentially be useful for occupational therapists to measure physical activity intensity levels.
\end{abstract}

Keyword: Perceived exertion; Monitoring; Office worker; Validity and reliability 